\title{
Front Matter: Volume 6996
}

, "Front Matter: Volume 6996," Proc. SPIE 6996, Silicon Photonics and

Photonic Integrated Circuits, 699601 (26 June 2008); doi: 10.1117/12.803012

SPIE. Event: SPIE Photonics Europe, 2008, Strasbourg, France 


\title{
PROCEEDINGS OF SPIE
}

\section{Silicon Photonics and Photonic Integrated Circuits}

\author{
Giancarlo C. Righini \\ Seppo K. Honkanen \\ Lorenzo Pavesi \\ Laurent Vivien \\ Editors
}

\section{7-10 April 2008 \\ Strasbourg, France}

Sponsored by

SPIE Europe

Cosponsored by

Alsace International (France)

Conseil Général du Bas-Rhin (France)

Région Alsace (France)

Communauté Urbaine de Strasbourg (France)

Cooperating Organizations

AFOP-Association Française des Industries de l'Optique et de la Photonique (France) • EOS-European Optical Society (Germany) • EPIC_European Photonics Industry Consortium (France) • ePIXnet

(Belgium) • IOP_Institute of Physics (United Kingdom) • NEMO-Network of Excellence on Micro-Optics (Belgium) • OLAS (Italy) • The OLLA Project (Germany) • OPERA 2015 (Belgium) • PhOREMOST (Ireland) Photonics Knowledge Transfer Network (United Kingdom) • Photonics Cluster (United Kingdom)

Photonics4Life (Germany) • Photonics 21 (Germany) • RhenaPhotonics Alsace (France)

Published by

SPIE 
The papers included in this volume were part of the technical conference cited on the cover and title page. Papers were selected and subject to review by the editors and conference program committee. Some conference presentations may not be available for publication. The papers published in these proceedings reflect the work and thoughts of the authors and are published herein as submitted. The publisher is not responsible for the validity of the information or for any outcomes resulting from reliance thereon.

Please use the following format to cite material from this book:

Author(s), "Title of Paper," in Silicon Photonics and Photonic Integrated Circuits, edited by Giancarlo C. Righini, Seppo K. Honkanen, Lorenzo Pavesi, Laurent Vivien, Proceedings of SPIE Vol. 6996 (SPIE, Bellingham, WA, 2008) Article CID Number.

ISSN 0277-786X

ISBN 9780819471949

Published by

SPIE

P.O. Box 10, Bellingham, Washington 98227-0010 USA

Telephone +1 3606763290 (Pacific Time) · Fax +1 3606471445

SPIE.org

Copyright (C) 2008, Society of Photo-Optical Instrumentation Engineers

Copying of material in this book for internal or personal use, or for the internal or personal use of specific clients, beyond the fair use provisions granted by the U.S. Copyright Law is authorized by SPIE subject to payment of copying fees. The Transactional Reporting Service base fee for this volume is $\$ 18.00$ per article (or portion thereof), which should be paid directly to the Copyright Clearance Center (CCC), 222 Rosewood Drive, Danvers, MA 01923. Payment may also be made electronically through CCC Online at copyright.com. Other copying for republication, resale, advertising or promotion, or any form of systematic or multiple reproduction of any material in this book is prohibited except with permission in writing from the publisher. The CCC fee code is 0277-786X/08/\$18.00.

Printed in the United States of America.

Publication of record for individual papers is online in the SPIE Digital Library.

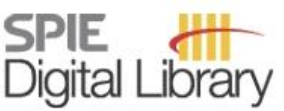

SPIEDigitalLibrary.org

Paper Numbering: Proceedings of SPIE follow an e-First publication model, with papers published first online and then in print and on CD-ROM. Papers are published as they are submitted and meet publication criteria. A unique, consistent, permanent citation identifier (CID) number is assigned to each article at the time of the first publication. Utilization of CIDs allows articles to be fully citable as soon they are published online, and connects the same identifier to all online, print, and electronic versions of the publication. SPIE uses a six-digit CID article numbering system in which:

- The first four digits correspond to the SPIE volume number.

- The last two digits indicate publication order within the volume using a Base 36 numbering system employing both numerals and letters. These two-number sets start with $00,01,02,03,04,05$, 06, 07, 08, 09, OA, OB ... 0Z, followed by 10-1Z, 20-2Z, etc.

The CID number appears on each page of the manuscript. The complete citation is used on the first page, and an abbreviated version on subsequent pages. Numbers in the index correspond to the last two digits of the six-digit CID number. 


\section{Contents}

xi Conference Committee

\section{SESSION 1 DEVICES INTEGRATION}

699604 Guided-wave electron optics for the integration of nanophotonic devices with nanoelectronic devices [6996-03]

J. Liñares, M. C. Nistal, V. Moreno, Univ. de Santiago de Compostela (Spain)

699605 Amorphous silicon optical waveguides and Bragg mirrors [6996-04]

A. Khanna, M. Mulot, Helsinki Univ. of Technology (Finland); S. Arpiainen, VTT Micro and Nanoelectronics (Finland); A. Säynätjoki, Helsinki Univ. of Technology (Finland); J. Ahopelto, VTT Micro and Nanoelectronics (Finland); S. Honkanen, H. Lipsanen, Helsinki Univ. of Technology (Finland)

\section{SESSION 2 PHOTONIC MATERIALS AND DEVICES}

699606 An organic semiconductor laser on silicon (Invited Paper) [6996-05]

A. E. Vasdekis, S. A. Moore, A. Ruseckas, T. F. Krauss, I. D. W. Samuel, G. A. Turnbull, Univ. of St. Andrews (United Kingdom)

699607 Preparation and characterization of $\mathrm{ZnO}$ particles embedded in organic-inorganic planar waveguide by sol-gel route [6996-06]

A. Chiappini, C. Armellini, A. Chiasera, M. Ferrari, Istituto di Fotonica e Nanotecnologie, CNR (Italy); R. Guider, Univ. di Trento (Italy); Y. Jestin, Istituto di Fotonica e Nanotecnologie, CNR (Italy); L. Minati, FBK-IRST (Italy); E. Moser, Univ. di Trento (Italy); G. N. Conti, S. Pelli, Nello Carrara Institute of Applied Physics, CNR (Italy); R. Retoux, ENSICAEN (France); G. C. Righini, Nello Carrara Institute of Applied Physics, CNR (Italy) and Dept. of Materials and Devices, CNR (Italy); G. Speranza, FBK-IRST (Italy)

699608 Cadmium telluride: a silicon-compatible optical material as an alternative technology for building all-optical photonic devices [6996-07]

A. Martínez, F. Cuesta-Soto, J. García, J. Martí, Univ. Politécnica de Valencia (Spain); N. V. Sochinskii, M. Abellan, J. Rodríguez-Fernández, Instituto de Microelectrónica de Madrid (Spain); S. Mengali, A. Mercuri, C. Corsi, Consorzio CREO (Italy); I. Reid, M. Robertson, Ctr. for Integrated Photonics (United Kingdom); S. Neretina, R. A. Hughes, J. Wojcik, J. S. Preston, P. Mascher, McMaster Univ. (Canada)

699609 Electron confinement effects in silver nanocluster embedded in sodalime glasses [6996-08] G. Speranza, L. Minati, Fondazione Bruno Kessler (Italy); A. Chiappini, A. Chiasera, CNR-IFN (Italy); C. Arfuso Duverger, LdOf, CNRS (France) and Univ. du Maine (France); M. Ferrari, CNR-IFN (Italy); G. C. Righini, CNR-IFAC (Italy) and Dept. of Materials and Devices, CNR (Italy) 
6996 OA Circular grating resonators as candidates for ultra-small photonic devices [6996-09]

S. Schönenberger, N. Moll, T. Stöferle, IBM Zürich Research Lab. (Switzerland); T. Wahlbrink, J. Bolten, AMO GmbH (Germany); S. Götzinger, ETH Zürich (Switzerland); T. Mollenhaver, C. Moormann, AMO GmbH (Germany); R. F. Mahrt, B. J. Offrein, IBM Zürich Research Lab. (Switzerland)

6996 OB Experimental verification of finesse enhancement scheme in two-ring resonator system [6996-10]

L. Y. M. Tobing, D. C. S. Lim, Nanyang Technological Univ. (Singapore); P. Dumon, R. Baets, Ghent Univ. (Belgium); M.-K. Chin, Nanyang Technological Univ. (Singapore)

6996 OD Analysis of silicon on insulator (SOI) optical microring add-drop filter based on waveguide intersections [6996-12]

A. Kaźmierczak, Multitel a.s.b.l. (Belgium); W. Bogaerts, D. Van Thourhout, Ghent Univ. (Belgium); E. Drouard, P. Rojo-Romeo, Univ. de Lyon, CNRS (France); D. Giannone, Multitel a.s.b.I. (Belgium); F. Gaffiot, Univ. de Lyon, CNRS (France)

6996 OE Active optical micro-resonators seen as mesoscopic "photonic atoms" [6996-13] Y. G. Boucher, ENIB/RESO Lab. (France); Y. Dumeige, P. Féron, ENSSAT/FOTON, CNRS (France)

\section{SESSION 4 DIAGNOSTIC AND CHARACTERISATION TECHNIQUES}

6996 OF Challenging nano-scale stress evaluation in glassy and crystalline semiconductor heterostructures (Invited Paper) [6996-14]

G. Pezzotti, Kyoto Institute of Technology (Japan); A. Leto, A. A. Porporati, M. Deluca, Kyoto Institute of Technology (Japan) and CNR Kyoto Research Ctr. (Japan); G. Righini, Dept. of Materials and Devices, CNR (Italy)

6996 OG Study of crystallization and phase mixing in $\mathrm{SiO}_{2} / \mathrm{SiO}_{x}$ superlattices through form birefringence measurements [6996-15]

M. Ghulinyan, MTLab (Italy); M. Wang, Zhejiang Univ. (China); A. Picciotto, G. Pucker, MTLab (Italy)

$6996 \mathrm{OH}$ Electro-optical properties of $\mathrm{BaTiO}_{3}-\mathrm{SrTiO}_{3}$ multilayer thin films for waveguide modulators [6996-16]

J. Hiltunen, M. Karppinen, P. Karioja, VTT Technical Research Ctr. of Finland (Finland); J. Lappalainen, J. Puustinen, V. Lantto, Univ. of Oulu (Finland); H. L. Tuller, Massachusetts Institute of Technology (USA)

$69960 \mathrm{~J}$ Advanced optical characterization of broad-band light-emitting color-center strip waveguides in lithium fluoride [6996-18]

R. M. Montereali, F. Bonfigli, M. Montecchi, E. Nichelatti, ENEA (Italy); M. Piccinini, INFN-LNF (Italy) 
6996 OL Implementation of quasi-phase-matching in semiconductor waveguides using whispering gallery mode resonators [6996-20]

Y. Dumeige, P. Féron, ENSSAT-FOTON, Univ. de Rennes 1, CNRS (France)

$69960 \mathrm{M}$ Compact spectrometer modelling based on wavelength-scale stationary wave Fourier transform in integrated optic [6996-21]

A. Morand, G. Custillon, P. Benech, Minatec (France); E. Le Coarer, Univ. Joseph Fourier, CNRS (France); G. Leblond, S. Blaize, Univ. de Technologie de Troyes, CNRS (France)

6996 ON Study and simulation for the sharp-corner of silicon-on-insulator waveguides [6996-22] D.-G. Sun, Univ. of Ottawa (Canada); X. Li, D. Wong, D\&T Photonics Corp. (Canada); T. Hall, Univ. of Ottawa (Canada)

699600 Bending loss, transition loss, mode coupling, and polarization coupling in bent waveguides [6996-23]

B. M. A. Rahman, D. M. H. Leung, City Univ. London (United Kingdom); S. S. A. Obayya, Univ. of Leeds (United Kingdom); K. T. V. Grattan, City Univ. London (United Kingdom)

\section{SESSION 6 WAVEGUIDES AND DEVICES I}

6996 OP Nested-ring Mach-Zehnder interferometer in silicon-on-insulator [6996-24]

S. Darmawan, Y. M. Landobasa, Nanyang Technological Univ. (Singapore); R. Baets, P. Dumon, Ghent Univ-IMEC (Belgium); M. K. Chin, Nanyang Technological Univ. (Singapore)

$69960 Q$ Broadband and highly efficient grating couplers for silicon-based horizontal slot waveguides [6996-25]

J. V. Galan, P. Sanchis, J. Blasco, J. Martí, Univ. Politécnica de Valencia (Spain)

6996 OR Echelle grating WDM (de-)multiplexers in SOI technology, based on a design with two stigmatic points [6996-26]

F. Horst, IBM Zürich Research Lab. (Switzerland); W. M. J. Green, IBM T.J. Watson Research Ctr. (USA); B. J. Offrein, IBM Zürich Research Lab. (Switzerland); Y. Vlasov, IBM T.J. Watson Research Ctr. (USA)

6996 OU Design of high gain $\mathrm{Er}^{3+}-\mathrm{Yb}^{3+}-\mathrm{Ce}^{3+} \mathrm{co}$-doped ZELA fluoride glass waveguide amplifier [6996-28]

F. Prudenzano, L. Allegretti, M. De Sario, L. Mescia, T. Palmisano, Politecnico di Bari (Italy); M. Ferrari, A. Chiasera, Y. Jestin, Institute of Photonics and Nanotechnology (Italy);

B. Boulard, O. Péron, Univ. du Maine, CNRS (France) 
6996 OV Quantum dot comb-laser as efficient light source for silicon photonics (Invited Paper) [6996-29]

A. Kovsh, A. Gubenko, I. Krestnikov, D. Livshits, S. Mikhrin, J. Weimert, Innolume GmbH (Germany); L. West, G. Wojcik, D. Yin, Innolume Inc. (USA); C. Bornholdt, N. Grote, Fraunhofer-Institut für Nachrichtentechnik Heinrich-Hertz-Institut (Germany); M. V. Maximov, A. Zhukov, St. Petersburg Physics and Technology Ctr. for Research and Education (Russia)

6996 OW Integration of photonics components in state-of-the-art CMOS technology (Invited Paper) [6996-30]

F. Lustenberger, D. Leipold, Espros Photonics AG (Switzerland)

6996 OY OIDA Silicon Photonics Alliance activities (Invited Paper) [6996-33]

M. Tlalka, T. Palkert, Luxtera, Inc. (USA)

\section{SESSION 8 PHOTONICS IN INDUSTRY II}

$69960 Z$ Challenges and innovations in very-large CCD and CMOS imagers for professional imaging (Invited Paper) [6996-34]

J. T. Bosiers, H. Stoldt, W. Klaassens, B. Dillen, I. Peters, E. Bogaart, DALSA Professional Imaging (Netherlands); R. Frost, DALSA Semiconductor (Canada); L. Korthout, J. Timpert, DALSA Professional Imaging (Netherlands)

$699610 \quad$ 10Gbit/s transceiver on silicon (Invited Paper) [6996-35]

J. Witzens, G. Masini, S. Sahni, B. Analui, C. Gunn, Luxtera, Inc. (USA); G. Capellini, Univ. Roma Tre (Italy)

699611 Silicon photonics in Pirelli (Invited Paper) [6996-36]

M. Romagnoli, L. Socci, L. Bolla, S. Ghidini, P. Galli, C. Rampinini, G. Mutinati, A. Nottola, A. Cabas, S. Doneda, M. Di Muri, R. Morson, T. Tomasi, G. Zuliani, S. Lorenzotti, D. Chacon, S. Marinoni, R. Corsini, F. Giacometti, S. Sardo, M. Gentili, G. Grasso, Pirelli Labs. s.p.a. (Italy)

699612 Low loss, high contrast planar optical waveguides based on low-cost CMOS compatible LPCVD processing (Invited Paper) [6996-37]

W. Hoving, XiO Photonics bv (Netherlands); R. Heideman, LioniX bv (Netherlands);

D. Geuzebroek, XiO Photonics bv (Netherlands); A. Leinse, LioniX bv (Netherlands); E. Klein,

R. Dekker, XiO Photonics bv (Netherlands)

\section{SESSION 9 ACTIVE WAVEGUIDES AND DEVICES I}

699613 Photonic switching on silicon: the FP6-Phologic approach (Invited Paper) [6996-38] J. Martí, A. Martínez, P. Sanchis, J. García, F. Cuesta-Soto, Univ. Politécnica de Valencia (Spain)

699614 Progress toward competitive Ge/Si photodetectors (Invited Paper) [6996-39] M. Morse, O. Dosunmu, T. Yin, Y. Kang, Intel Corp. (USA); G. Sarid, E. Ginsburg, R. Cohen, M. Zadka, Intel Electronics Ltd. (Israel) 
699615 Carrier-depletion-based optical modulator integrated in a lateral structure in a SOI waveguide [6996-40]

D. Marris-Morini, L. Vivien, Univ. Paris Sud, CNRS (France); J. M. Fédéli, Minatec (France);

E. Cassan, G. Rasigade, X. Le Roux, Univ. Paris Sud, CNRS (France); P. Lyan, Minatec

(France); S. Laval, Univ. Paris Sud, CNRS (France)

699616 Integrated microdisk based lasers for electro-optical applications in CMOS compliance [6996-41]

F. Mandorlo, Univ. de Lyon, CNRS (France) and Minatec (France); P. Rojo Romeo,

$X$. Letartre, Univ. de Lyon, CNRS (France); J.-M. Fedeli, Minatec (France); P. Regreny, Univ. de Lyon, CNRS (France); P. Grosse, Minatec (France); P. Viktorovitch, Univ. de Lyon, CNRS (France)

699618 Optimization of $\mathrm{Al}_{2} \mathrm{O}_{3}: \mathrm{Er}^{3+}$ waveguide technology for active integrated optical devices [6996-43]

K. Wörhoff, J. Bradley, F. Ay, D. Geskus, T. Blauwendraat, M. Pollnau, Univ. of Twente (Netherlands)

699619 Further improvements in $\mathrm{Er}^{3+}$ coupled to Si nanoclusters rib waveguides [6996-44] A. Pitanti, D. Navarro-Urrios, R. Guider, N. Daldosso, Univ. di Trento (Italy); F. Gourbilleau, L. Khomenkova, R. Rizk, Ecole Nationale Supérieure d'Ingenieurs de Caen et Ctr. de Recherche, CNRS (France); L. Pavesi, Univ. di Trento (Italy)

6996 1A Mode-locked fiber/waveguide lasers based on a fiber taper embedded in carbon nanotubes/polymer composite [6996-45]

Q. Wang, College of Optical Sciences, Univ. of Arizona (USA); K. Khanh, Cornell Univ. (USA); S. Honkanen, College of Optical Sciences, Univ. of Arizona (USA) and Helsinki Univ. of Technology (Finland); F. Kueppers, College of Optical Sciences, Univ. of Arizona (USA)

6996 1B Evanescent multimode longitudinal pumping scheme for Si-nanocluster sensitized $\mathrm{Er}^{3+}$ doped waveguide amplifiers [6996-46]

V. Toccafondo, S. Faralli, F. Di Pasquale, Scuola Superiore Sant'Anna (Italy); J. Carreras,

B. Garrido, Univ. de Barcelona (Spain)

\section{SESSION 11 ADVANCED PROCESSES AND DEVICES}

6996 IC Si/SiGe bound-to-continuum quantum cascade terahertz emitters (Invited Paper) [6996-47] D. J. Paul, Univ. of Glasgow (United Kingdom); G. Matmon, Univ. of Cambridge (United Kingdom); L. Lever, Z. Ikonić, R. W. Kelsall, Univ. of Leeds (United Kingdom); D. Chrastina, G. Isella, H. von Känel, L-NESS (Italy)

6996 1D Building technology plafforms and foundries for photonic integrated circuits in Europe (Invited Paper) [6996-48]

R. Baets, P. Dumon, W. Bogaerts, Ghent Univ. - IMEC (Belgium); A. Chelnokov, J.-M. Fedeli, Minatec (France); L. Vanholme, D. Steyaert, Ghent Univ. - IMEC (Belgium)

6996 IE Modelling of silicon and active photonic integrated circuits (Invited Paper) [6996-49]

D. F. G. Gallagher, T. Felici, Photon Design (United Kingdom) 
$69961 G$ Optical buffering scheme based on two-ring resonator system [6996-51]

L. Y. M. A. L. Tobing, M.-K. Chin, Nanyang Technological Univ. (Singapore)

6996 1H Design of a reconfigurable optical interconnect for large-scale multiprocessor networks [6996-52]

I. Artundo, Vrije Univ. Brussel (Belgium); W. Heirman, Ghent Univ. (Belgium); C. Debaes, Vrije Univ. Brussel (Belgium); J. Dambre, J. Van Campenhout, Ghent Univ. (Belgium);

H. Thienpont, Vrije Univ. Brussel (Belgium)

699611 Performance of a free space optics subsystem boosted by SCM implementation [6996-53] D. V. N. Coelho, T. V. N. Coelho, M. T. M. R. Giraldi, Instituto Militar de Engenharia (Brazil); M. J. Pontes, M. E. V. Segatto, Federal Univ. of Espírito Santo (Brazil); J. C. W. A. Costa, Univ. Federal do Pará (Brazil)

$69961 \mathrm{~J}$ A novel integrated optical device for spectral coding in OCDMA networks [6996-54] A. V. Shamray, A. S. Kozlov, I. V. llichev, M. P. Petrov, loffe Physico-Technical Institute (Russia)

\section{SESSION 13 WAVEGUIDES AND DEVICES II}

6996 1L Phase matched magnetooptic planar waveguide elaborated by magnetic nanoparticles embedded in an organic-inorganic sol-gel matrix [6996-56]

F. Choveikani, F. Royer, D. Jamon, Univ. Jean Monnet (France); S. Neveu, Univ. Pierre et Marie Curie (France); J. Charara, Univ. Libanaise (Lebanon)

699610 Slow-light photonic crystal waveguides with ring-shaped holes on silicon-on-insulator [6996-59]

A. Säynätjoki, Helsinki Univ. of Technology (Finland); M. Mulot, Helsinki Univ. of Technology (Finland) and VTT Micro and Nanoelectronics (Finland); K. Vynck, D. Cassagne, Univ. Montpellier II, CNRS (France); J. Ahopelto, VTT Micro and Nanoelectronics (Finland); H. Lipsanen, Helsinki Univ. of Technology (Finland)

\section{POSTER SESSION}

6996 IP Application of discrete layer peeling algorithm for design of selective photonic devices obeying the constraints of the fabrication process [6996-60] M. Wielichowski, S. Patela, Wroclaw Univ. of Technology (Poland)

6996 IR Quantization of coupled waveguided modes progression in integrated photonic devices [6996-62]

J. Liñares, M. C. Nistal, D. Barral, V. Moreno, C. Montero, Univ. de Santiago de Compostela (Spain)

6996 is Wide tunable thermo-optical filters with polymer micro-ring resonators [6996-63] A. Maalouf, D. Bosc, P. Grosso, M. Gadonna, M. Thual, F. Henrio, S. Haesaert, Ecole Nationale Supérieure des Sciences Appliquées et de Technologie (France) and Telecom Bretagne (France) 
6996 1T A design method for a ratiometric wavelength monitor using a pair of directional couplers acting as edge filters [6996-64]

A. M. Hatta, Dublin Institute of Technology (Ireland); Q. Wang, Data Storage Institute (Singapore); G. Farrell, Dublin Institute of Technology (Ireland); J. Zheng, Jilin Univ. (China)

6996 IV A new method for solving nonhermitian perturbation theory for the microdisc resonators with phi-periodic refractive index [6996-66]

S. Keyvaninia, M. Karvar, A. Bahrampour, Sharif Univ. of Technology (Iran) and International Ctr. of Science, High Technology, and Environmental Science (Iran)

6996 IW Studies of different modulation techniques in the integration of SCM optical communication system [6996-67]

D. V. N. Coelho, M. T. M. R. Giraldi, Instituto Militar de Engenharia (Brazil); D. J. C. Coura, M. E. V. Segatto, M. J. Pontes, Federal Univ. of Espírito Santo (Brazil); J. C. W. A. Costa, Univ. Federal do Pará (Brazil)

6996 1X Dynamic behavior of electric field in the microrings in the presence of Kerr and two-photon absorption [6996-68]

S. Keyvaninia, M. Karvar, A. Bahrampour, Sharif Univ. of Technology (Iran) and International Ctr. of Science, High Technology, and Environmental Science (Iran)

$69961 \mathrm{Z}$ Electro-tuning of the photonic band gap in SOI-based structures infiltrated with liquid crystal [6996-70]

V. A. Tolmachev, S. A. Grudinkin, Trinity College (Ireland) and loffe Physico-Technical Institute (Russia); J. A. Zharova, loffe Physico-Technical Institute (Russia); V. A. Melnikov, Trinity College (Ireland); E. V. Astrova, loffe Physico-Technical Institute (Russia); T. S. Perova, Trinity College (Ireland)

Author Index 
Downloaded From: https://www.spiedigitallibrary.org/conference-proceedings-of-spie on 26 Apr 2023

Terms of Use: https://www.spiedigitallibrary.org/terms-of-use 


\title{
Conference Committee
}

\author{
Symposium Chairs
}

Hugo Thienpont, Vrije Universiteit Brussel (Belgium)

Patrick P. Meyrueis, Université Louis Pasteur (France)

Giancarlo C. Righini, Istituto di Fisica Applicata Nello Carrara (Italy)

\section{Conference Chairs}

Giancarlo C. Righini, Istituto di Fisica Applicata Nello Carrara (Italy)

Seppo K. Honkanen, Helsinki University of Technology (Finland)

Lorenzo Pavesi, Università degli Studi di Trento (Italy)

Laurent Vivien, Université Paris-Sud II, CNRS (France)

Program Committee

Gonçal Badenes, Institut de Ciències Fotòniques (Spain)

Michel J. F. Digonnet, Stanford University (USA)

Alfred Driessen, University of Twente (Netherlands)

Louay A. Eldada, DuPont Photonics Technologies (USA)

Jean-Marc Fédéli, CEA-LETI (France)

Mário F. S. Ferreira, Universidade de Aveiro (Portugal)

Gerard Guillot, Institut National des Sciences Appliquées de Lyon (France)

Kazuoki Hirao, Kyoto University (Japan)

Andrea Irace, Università degli Studi di Napoli Federico II (Italy)

Bahram Jalali, University of California, Los Angeles (USA)

El-Hang Lee, Inha University (South Korea)

Michael Morse, Intel Corporation (USA)

Santiago Miguel Olaizola, Centro de Estudios e Investigaciones

Técnicas de Gipuzkoa (Spain)

Thomas P. Pearsall, European Photonics Industry Consortium (France)

Stefano Pelli, Istituto di Fisica Applicata Nello Carrara (Italy)

Juha T. Rantala, Silecs Oy (Finland)

Graham T. Reed, University of Surrey (United Kingdom)

Marco Romagnoli, Pirelli \& C. S.p.A. (Italy)

Alberto Scandurra, STMicroelectronics (Italy)

Ali Serpengüzel, Koç University (Turkey)

Herbert Venghaus, Fraunhofer-Institut für Nachrichtentechnik Heinrich-

Hertz-Institut (Germany) 
Session Chairs

1 Devices Integration

Giancarlo C. Righini, Istituto di Fisica Applicata Nello Carrara (Italy)

2 Photonic Materials and Devices

Seppo K. Honkanen, Helsinki University of Technology (Finland)

3 Microresonators

Stefano Pelli, Istituto di Fisica Applicata Nello Carrara (Italy)

$4 \quad$ Diagnostic and Characterisation Techniques

Ali Serpengüzel, Koç University (Turkey)

$5 \quad$ Modelling and Design

Dominic F. G. Gallagher, Photon Design (United Kingdom)

$6 \quad$ Waveguides and Devices I

Javier Martí, Universidad Politécnica de Valencia (Spain)

$7 \quad$ Photonics in Industry

Thomas P. Pearsall, European Photonics Industry Consortium (France)

$8 \quad$ Photonics in Industry II

Laurent Vivien, Université Paris-Sud-XI (France)

9 Active Waveguides and Devices I

El-Hang Lee, Inha University (South Korea)

10 Active Waveguides and Devices II

El-Hang Lee, Inha University (South Korea)

11 Advanced Processes and Devices

Lorenzo Pavesi, Università degli Studi di Trento (Italy)

12 Photonics in Telecommunication Systems

Lorenzo Pavesi, Università degli Studi di Trento (Italy)

13 Waveguides and Devices II

Seppo K. Honkanen, Helsinki University of Technology (Finland) 